\title{
Mobiles de l'Intention Entrepreneuriale : Cas des Etudiant(e)s Universitaires de la Région Rabat -Salé-Kenitra du Maroc
}

\author{
Mustapha Achibane, Professeur Habilité \\ Ghyzlane Andaloussi, Doctorante en Sciences de Gestion \\ Ecole Nationale de Commerce et de Gestion de Kenitra
}

Doi:10.19044/esj.2019.v15n19p259 URL:http://dx.doi.org/10.19044/esj.2019.v15n19p259

\section{Résumé}

La recherche en entrepreneuriat a connu une évolution qui s'est accompagnée par une croissance de l'intérêt accordé à l'entrepreneur et à la recherche des facteurs de réussite, qui expliquent pourquoi certains individus parviennent à franchir le cap de création d'entreprise tandis que d'autres n'y arrivent pas. Les recherches ont pendant longtemps supposé que les caractéristiques personnelles de l'individu-créateur étaient à l'origine de ces différences (D’Amboise, 1989). Suite aux premières recherches en entrepreneuriat, le phénomène entrepreneurial est expliqué principalement par le caractère du créateur. L'avènement des approches processuelles dans le champ de l'entrepreneuriat a favorisé les études qui se posent la question du «pourquoi » et du « comment» (Fayolle, 2002). Dans cette perspective, nous allons nous intéresser à l'amont du processus entrepreneurial, afin de mieux appréhender les mobiles à l'origine de l'intention entrepreneuriale. Dans la présente étude, nous allons mener sur le terrain une étude exploratoire à travers un questionnaire élaboré à partir de la revue de la littérature sur l'intention entrepreneuriale. Nous nous sommes appuyés sur un échantillon composé de 150 étudiant(e)s du troisième cycle $(\mathrm{Bac}+3$ et $\mathrm{Bac}+5)$, qui sont considérés le plus susceptibles à s'orienter vers la création d'entreprise à la fin de leurs formations. Aussi, nous nous sommes basés sur la théorie du comportement planifié de Ajzen (1991), qui considère la désirabilité de l'exercice entrepreneurial comme principal déterminant qui influence la concrétisation de l'activité entrepreneuriale.

Mots-clés: Entrepreneuriat, Intention entrepreneuriale, Etudiants universitaires, Théorie du comportement planifié 


\title{
Mobiles of Entrepreneurial Intention: The Case of University Students in the Rabat-Sale-Kenitra Region of Morocco
}

\author{
Mustapha Achibane, Professeur Habilité \\ Ghyzlane Andaloussi, Doctorante en Sciences de Gestion \\ Ecole Nationale de Commerce et de Gestion de Kenitra
}

\begin{abstract}
Entrepreneurial research has evolved along with growth in entrepreneurial interest and the search for success factors. This explains why some individuals can make it through the process of creating and starting their own companies while others cannot. For a long time, research has assumed that the personal characteristics of the founder were the main origin of these differences (D'Amboise, 1989). Following the first pieces of research in entrepreneurship, the entrepreneurial phenomenon is mainly explained by the character of the "founder". The advent of procedural approaches in the field of entrepreneurship has endorsed the study of the following questions of "why" and "how" (Fayolle, 2002). This paper focuses on the upstream of the entrepreneurial process, in order to better capture the mobile aspects at the origin of the entrepreneurial intention. An exploratory study was conducted in the field through a questionnaire developed from the literature review on entrepreneurial intention. In addition, a sample of 150 students was used from the third cycle $(\mathrm{Bac}+3$ and $\mathrm{Bac}+5)$. This refers to those who are considered to be the most likely to move towards the foundation of a company at the end of their training. Also, we based ourselves on the theory of planned behaviour of Ajzen (1991), which considers the desirability of the entrepreneurial exercise as the main determinant that influences the implementation of entrepreneurial activity.
\end{abstract}

Keywords: Entrepreneurship, Entrepreneurial intention, University Students, Theory of Planned Behavior

\section{Introduction}

Pour Julien et Marchesnay (1996), la notion d'entrepreneuriat dans la littérature nous renvoie vers trois concepts: l'entrepreneur, l'esprit d'entreprise (esprit d'entreprendre) et la création d'entreprise. On retient alors, que le phénomène entrepreneurial a toujours été défini selon diverses 
approches privilégiant ainsi les résultats de l'action d'entreprendre, les caractéristiques des individus agissant en tant qu'entrepreneurs et enfin, les processus et les stratégies conduisant au comportement.

Toutefois, la phase amont de la création d'entreprise à savoir l'intention d'entreprendre, a longtemps été négligée au niveau de la recherche en entrepreneuriat. Cette phase s'insère entre le processus de découverte et le processus d'exploitation, (Davidsson, 2006). Dès lors l'enjeu est d'appréhender le passage de l'intention vers l'acte de création d'entreprise.

L'objectif de notre contribution est de dresser un état de l'art du concept de l'intention entrepreneuriale, et de discuter autour des mobiles qui l'influencent et mènent vers la concrétisation de l'exercice entrepreneurial.

Pour mener à bien cette discussion, nous allons dans un premier temps, recenser le cadre théorique de l'intention entrepreneuriale, et les facteurs nécessaires à la faisabilité de l'exercice entrepreneurial. Dans un second temps, dans le cadre d'une étude exploratoire nous chercherons à mieux comprendre le concept d'intention, par sa vérification auprès d'un échantillon des jeunes étudiants des grandes écoles et universités marocaines situés dans la région Rabat-Salé-Kenitra. Au travers cette étude, nous essayerons de comprendre l'origine de la volonté et de l'enthousiasme à prendre des décisions en vue de la création d'entreprise.

\section{Approche théorique de l'intention entrepreneuriale}

Pendant ces dernières décennies, plusieurs auteurs ont tenté d'expliquer le phénomène entrepreneurial, spécialement à travers l'exploration des mobiles et facteurs qui favorisent la création d'entreprise. Ainsi, certains facteurs ont été mis en avant, à savoir les traits de personnalité, et les caractéristiques démographiques.

Parallèlement, certains auteurs se sont intéressés davantage aux approches processuelles du phénomène entrepreneurial (identification d'opportunité, émergence organisationnelle, vision entrepreneuriale, projet d'entreprendre, etc.). A cet égard, la phase intentionnelle du processus entrepreneurial mérite qu'on s'y intéresse davantage.

Le modèle de Learned (1992) propose un processus de formation qui aboutit in fine à la décision de créer ou non une entreprise. Ce modèle, comporte trois dimensions du processus de création, à savoir :

La propension de créer : certains individus ont une combinaison de caractéristiques psychologiques et un passé professionnel qui les rendent plus susceptibles d'essayer de créer une affaire que d'autres ;

$>\quad$ L'intention de créer : parmi ceux-ci certains rencontrent des situations qui déclenchent l'intention de créer ; 
La structuration des informations : cette étape consiste à rechercher et à trouver dans l'environnement les informations significatives, et à en déduire les tâches à exécuter pour créer l'organisation.

Ce modèle processuel décortique l'intention entrepreneuriale dans un environnement social en une vision qui se traduit en une intention suite à la détection d'une opportunité, pour la concrétiser en une décision de création d'entreprise. Ainsi, on s'accorde sur le fait que le passage à l'acte entrepreneurial est un processus qui dépend de la vision que se fait l'individu de son future, et de l'intensité de son intention d'agir, (Moreau \& Raveleau, 2006). A travers la revue de la littérature, on a pu retenir diverses approches de la vision entrepreneuriale et parmi elles, on retient la définition de Benavent et Verstraete (2000) qui stipulent : « pour choisir une orientation, le dirigeant doit d'abord s'être fait une image mentale d'un état futur possible et souhaitable de l'organisation. »

Ensuite, nous revenons sur le concept d'intention comme étant indispensable à la création d'entreprise. Ajzen (1987) caractérise l'intention de se comporter d'une certaine façon, comme une structure cognitive, incluant à la fois les fins et les moyens. La revue de la littérature nous permet de relever différents essais de définition. On note que Bird (1988, p.445) a considéré l'intention comme « un processus qui naît avec les besoins, les valeurs, les habitudes et les croyances de l'individu » Tounès (2003, p.58) vient appuyer la définition proposée par Bird en ajoutant que «l'intention est, certes, une volonté individuelle qui s'inscrit dans un processus cognitif mais elle est fonction des contextes socioculturel et économique ». Davidsson (2006) précise aussi que c'est une manifestation mentale qui implique une volonté, dont le but est la création d'entreprise.

L'intention entrepreneuriale revêt alors, un caractère évolutif qui dépend des facteurs contextuels et plus généralement de l'environnement dans lequel s'inscrit l'acteur de l'action (Tounès, 2003). Vient ensuite la décision de créer l'entreprise, qui est considérée par Hernandez et Marco (2006) comme un processus de détection et d'exploitation d'opportunité. C'est le maillon manquant qui explique le décalage entre ceux qui ont l'intention d'entreprendre sans forcément passer à l'acte, et ceux qui le font réellement. Ainsi, tout commence par le déclenchement du processus entrepreneurial, lors duquel, l'individu envisage sérieusement d'entreprendre. Il manifeste une intention qui correspond à ses aspirations (désirabilité) et il doit avoir les aptitudes nécessaires pour réaliser son projet (faisabilité). L'individu entame alors la phase d'engagement avant la concrétisation de l'acte de création. Ensuite, le processus étant lancé, il se consacre à la mise en œuvre de son projet.

La dimension temporelle de l'intention est primordiale pour comprendre la relation entre l'intention entrepreneuriale et le passage à l'acte. 
L'intensité de l'intention peut varier considérablement avec le temps. Au terme de leur recherche, Moreau et Raveleau (2006) définissent l'intention entrepreneuriale comme un processus non linéaire, composé de séquences : formation, développement, maintien, déclin et aboutissement. Les variables se différent selon la phase étudiée. Dans une démarche inductive, les auteurs interrogent 210 étudiants en sciences de gestion et d'économiques inscrits en Master 1 et 2 à la Faculté des sciences économiques et de gestion de Nantes (France). Les auteurs montrent qu'une intention peut connaître des évolutions différentes d'une personne à l'autre et «que c'est un état de pensée en mouvement qui s'étire sur un laps de temps relativement long, et qui peut connaître des fluctuations rapides d'intensité (en moyenne tous les deux ans) » (Moreau \& Raveleau, 2006, p.127).

\section{Mobiles expliquant l'intention entrepreneuriale au cour du processus de création d'entreprise}

La revue des théories sur le comportement intentionnel, a permis de comprendre le fonctionnement et l'agencement des variables expliquant l'intention entrepreneuriale et de préciser la place occupée au cœur du processus d'émergence entrepreneuriale (Emin, 2003).

Figure 1. Différentes phases du processus entrepreneurial

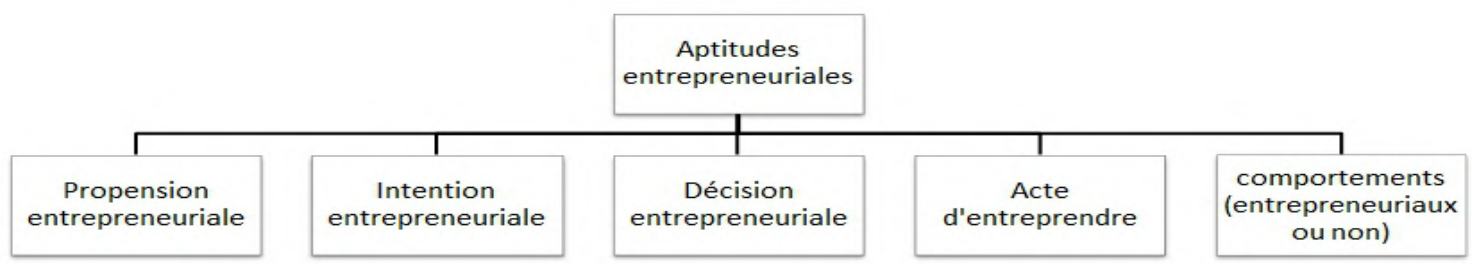

Boyer (1997, p.268), Les limites de la rationalité : rationalité, éthique et cognition.

On retient de cette figure, que le processus entrepreneurial est un cheminement d'étapes qui requièrent des aptitudes entrepreneuriales. Ces aptitudes entrepreneuriales peuvent s'enrichir au fur et à mesure que l'individu progresse au sein du processus. Il peut chercher à augmenter ses aptitudes entrepreneuriales par le suivi d'enseignements ou de formation. La propension entrepreneuriale signifie que sous l'influence de son environnement, notamment la famille, les proches, la formation, et des propres expériences entrepreneuriales, l'individu est sensibilisé à l'entrepreneuriat et n'écarte pas l'éventualité de fonder son entreprise. La propension peut se convertir en une intention entrepreneuriale par l'existence d'une idée ou d'un projet d'affaire plus ou moins formalisé, et l'engagement personnel. La décision implique que l'individu est conforté dans son intention; la formalisation de l'idée ou du projet est accomplie, ensuite les ressources de différentes natures (financières 
et logistiques) sont globalement mobilisées. L'acte d'entreprendre correspond au démarrage physique de l'activité qui se manifeste par la réalisation des premiers produits ou services.

Par ailleurs, il est indispensable d'aborder les trois étapes constituant l'intention entrepreneuriale: la première consiste en la formation d'une vision, qui correspond à une représentation mentale de la création de l'entreprise, de ses activités et de son environnement, comme futur possible. La deuxième étape est la transformation de cette vision en une véritable intention de création. Selon le contexte entrepreneurial, la vision correspond au démarrage $\mathrm{du}$ processus entrepreneurial. La troisième étape consiste en la prise de décision ; celle-ci déclenche l'action et finit par la réalisation de l'intention, c'est-à-dire à la mobilisation des moyens nécessaires pour concrétiser le but recherché. Pour que l'action soit effectivement déclenchée, l'intention doit se transformer en une décision d'action.

Aussi d'aborder les facteurs psychologiques et contextuels de l'individu-créateur qui favorisent le processus de prise de décision présenté par Shapero (1975):

$>\quad$ La discontinuité (variable de situation) : Met en exergue les situations négatives (Pushes) et les situations positives (pulls);

$>\quad$ La crédibilité de l'acte (variable sociologique) : Est issue de l'image d'imitation et de la culture entrepreneuriale;

$>\quad$ La faisabilité (variable économique) : Dépend essentiellement de 6 types de ressources (Money, Men, Machines, Materials, Market, Management).

Selon Belley (1989), il manque une variable pertinente. Il propose d'intégrer au modèle de Shapero la variable de l'opportunité, comme la vision de l'entrepreneur qui va permettre de «présenter le potentiel d'exploitation d'une idée pour en tirer profit» (Hernandez, 2001, p.71).

La théorie de Ajzen (1991) vient illustrer au mieux le modèle de l'intention entrepreneuriale, et présente les trois déterminants conceptuels favorisant l'acte de création d'entreprise, à savoir les attitudes associées au comportement, les perceptions du contrôle comportemental et les normes subjectives. Les deux premiers éléments concourent à l'attractivité du comportement et peuvent être rapprochés de la notion de désirabilité. Tandis que le troisième élément peut être rapproché des concepts de faisabilité et d'efficacité personnelle. 
Figure 2. Modélisation théorique de l'intention entrepreneuriale

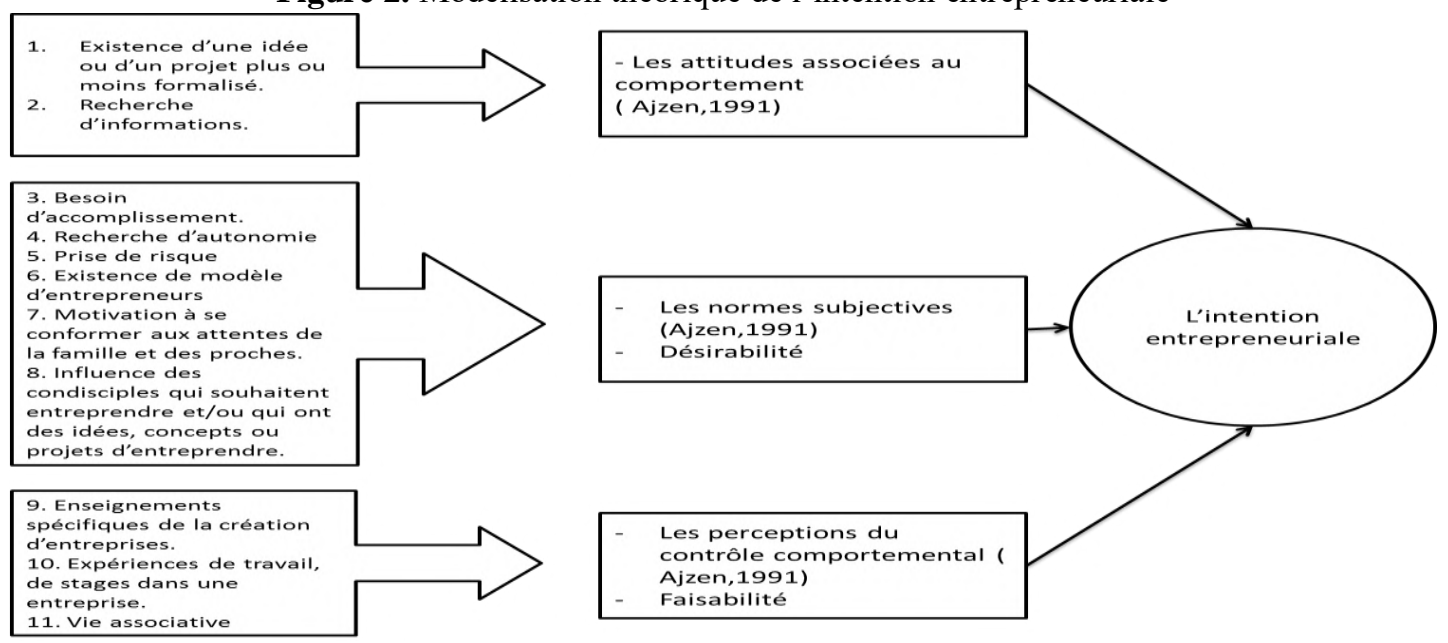

Tounès (2006, p.48), L’intention entrepreneuriale des étudiants : le cas français.

Faisant référence au modèle de Ajzen, on note que le processus de création est la transformation de l'intention en action. Il correspond à la démarche qui conduit l'individu à créer effectivement une nouvelle entreprise. Cet engagement aboutit à une irréversibilité : son arrêt est considéré comme un échec, sa continuité une escalade de l'engagement. Toutefois, le lien entre intention et action n'est ni immédiat ni certain. Plus précisément, la formation d'une intention entrepreneuriale dépend de la perception de la désirabilité, la propension à agir et la perception de la faisabilité du comportement (Shapero \& Sokol, 1982). Ceci dit, l'engagement est l'élément clé du processus de passage à l'acte. Le passage à l'acte requiert l'intervention de plusieurs facteurs qui renforcent ou inhibent le passage à l'acte. Les traits de personnalité, comme le concept de soi et l'orientation de l'esprit conditionnent la formation de plans d'action pour la concrétisation des intentions.

L'effet du temps sur les intentions dépend, d'une part, de l'intensité de l'intention initiale et, d'autre part, des prédispositions individuelles à changer ses intentions. Outre le facteur temps, l'acte d'entreprendre pourrait être affecté positivement (facilité) ou négativement (bloqué) par l'occurrence d'évènement imprévus. Il s'agit de l'apparition d'évènements précipitatifs qui correspondent à des facteurs contextuels agissant sur l'individu. Ces derniers pourraient s'avérer comme des contraintes ou au contraire comme des incitateurs à l'acte entrepreneurial. Néanmoins, il existe des forces qui appartiennent à l'environnement immédiat de l'entrepreneur, reliées soit à la vie personnelle, soit à la vie professionnelle, et qui présentent des aspects positifs ou négatifs.

Toutefois, il convient de comprendre et d'analyser les mobiles qui se manifestent chez les individus au cours du processus entrepreneurial amont. 
Ce qui nous renseigne sur certaines variables motivationnelles et situationnelles qui peuvent influencer l'intention, et éventuellement l'acte de création. Les mobiles sont inhérents à l'individu et conformes à sa personnalité, à ses intérêts et parfois à son projet d'entreprise. Ils sont de différentes natures et présentent une importance variable.

$>\quad$ Les mobiles économiques : Entreprendre serait profiter des occasions de faire des bénéfices que d'autres auraient négligées. Aujourd'hui, tous les auteurs intervenant dans le champ de l'entrepreneuriat s'accordent à dire que le mobile économique n'est pas le seul qui détermine le processus entrepreneurial amont ;

$>\quad$ Les mobiles psychologiques : Schumpeter (1935) recense plusieurs mobiles qui peuvent expliquer l'acte entrepreneurial (le rêve, la volonté de bâtir un empire, le sentiment de puissance et de propriété, la volonté d'élévation sociale, diriger une entité économique). Cependant, la reconnaissance des mobiles individuels ne doit pas conduire à négliger le poids des contraintes et des influences sociales et culturelles dans le processus entrepreneurial, notamment dans ses phases intentionnelles et d'acte de création ;

$>\quad$ Les mobiles socioculturels : Les conditions socioculturelles constituent « le moule » dans lequel sont coulées les capacités de chacun. Si le capital financier est indispensable à toute activité entrepreneuriale, la culture et la religion fournissent le capital «spirituel ». la famille a toujours été le principal agent de socialisation (Vallerand, 1994). Le réseau primaire des connaissances de l'individu prépare souvent le terrain à une carrière d'entrepreneur. Il inclut globalement la famille, les amis, les connaissances scolaires et professionnelles. Bien qu'il fournisse des informations plus générales, le caractère personnel et informel de ce réseau fait qu'il est le premier et le plus souvent consulté (Raijman, 2001).

En outre, il convient de souligner aussi l'importance des facteurs contingents de l'intention entrepreneuriale, à savoir l'expérience professionnelle, l'ancrage territorial et les systèmes d'appui et de soutien à la création d'entreprise.

L'expérience professionnelle : Le travail constitue souvent un lieu de rencontre essentiel entre le créateur et l'idée. Il permet d'observer et d'explorer en profondeur un secteur d'activité, un marché et un métier. L'expérience professionnelle peut augmenter les perceptions des aptitudes entrepreneuriales et de ce fait, contribuer éventuellement, au sein du processus entrepreneurial, à la formation de l'intention et à l'acte de création ; 
L'ancrage territorial: Le territoire est un pôle de ressources dont l'attractivité entrepreneuriale est indéniable. Il peut développer les perceptions de disponibilité des ressources nécessaires qui influenceraient le processus entrepreneurial amont, notamment dans ses phases intentionnelle et d'acte de création ;

$>\quad$ Les systèmes d'appui et de soutien à la création d'entreprise : Mis en place par les pouvoirs publics pour favoriser la création d'entreprise, ces systèmes sont des moyens d'actions qui fournissent les ressources nécessaires susceptibles de faciliter, le long du processus entrepreneurial, le parcours des détenteurs de projets. Ces systèmes sont des facteurs contingents qui peuvent influencer favorablement les perceptions de disponibilité des ressources, qui à leur tour, renforceront l'intention voire conduiront à la concrétisation de l'acte de création.

\section{Méthodologie de recherche}

La recherche sur l'enseignement et l'entrepreneuriat n'est pas un fait nouveau. La première recherche traitant l'intention entrepreneuriale d'une population d'étudiants en gestion remonte à 1975, elle est à l'actif de Brockhaus (1975). La formation en entrepreneuriat, notamment pour des étudiants de 3ème cycle, intervient à un moment où ils doivent souvent décider de leur carrière. Elle joue un rôle capital dans leur choix professionnel et leur offre un cadre où peuvent s'intégrer de nouvelles attitudes, aptitudes et perceptions.

Dans ce même cadre, on va essayer dans ce travail à comprendre et à expliquer les variables personnelles et contextuelles, agissant sur l'intention entrepreneuriale des étudiants. Ainsi, pour atteindre l'objectif de notre travail, nous avons choisi de mener une étude exploratoire auprès d'une population cible composée des étudiants du troisième cycle $(\mathrm{Bac}+3$ et $\mathrm{Bac}+5)$, puisqu'ils sont à quelques mois d'entamer leur vie professionnelle et manifestent une variété d'intentions de carrière. Pour la taille de notre échantillon (150 étudiant(e)s), nous avons opté pour la méthode d'échantillonnage par convenance. Le choix de cette méthode peut être considérer comme l'une des limites de cette recherche, vu la non disponibilité d'une base de sondage pour pouvoir utiliser une méthode d'échantillonnage représentative. 
Figure 3. Répartition des interviewées en fonction du sexe, de l'âge et du niveau d'étude
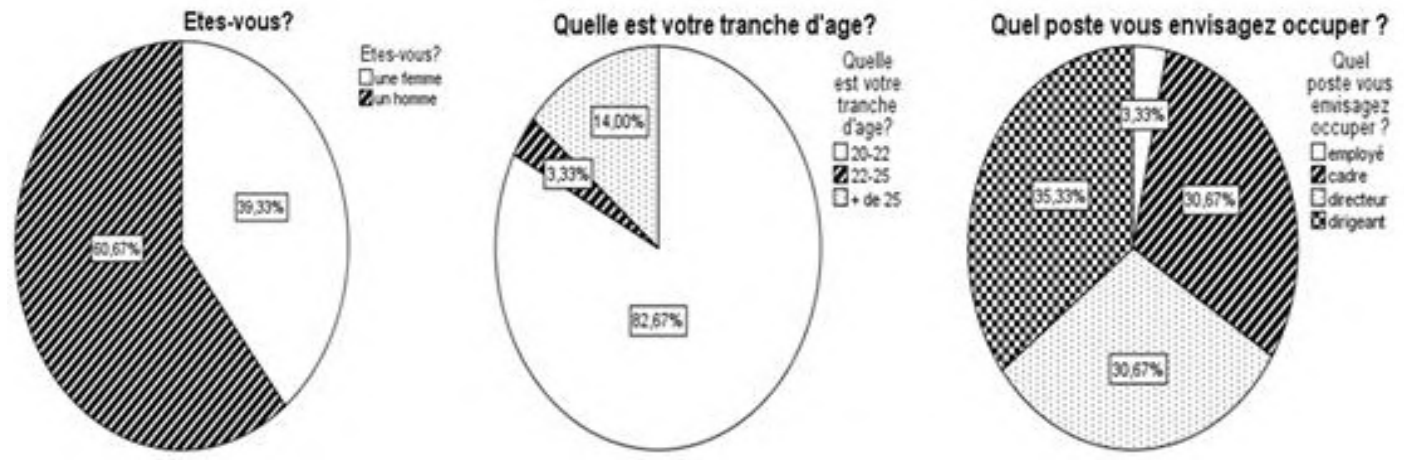

Dans le questionnaire (en annexe), les perceptions sont majoritairement traduites en questions qualitatives à choix multiples majoritairement. Des échelles de Likert à 5 points ont été utilisées pour mesurer l'intention, la désirabilité et la faisabilité perçue. Les échelles allaient de « pas du tout d'accord »à « tout à fait d'accord ». Le traitement des données a été réalisé avec le logiciel d'analyse de données SPSS.

\section{La présentation et discussion des résultats}

La décision de démarrer une nouvelle entreprise est supposée être planifiée pendant un certain temps, toutefois, certains facteurs impulsent cette décision. Afin de mieux évaluer l'intention entrepreneuriale des étudiants interviewés, nous allons nous baser sur la théorie du comportement planifié de Ajzen (1991).

Suite à notre étude, nous constatons dans un premier temps, que notre échantillon est favorable à l'entrepreneuriat à raison de $84.7 \%$, et que $70 \%$ des étudiants sondés pensent qu'entreprendre est un futur proche, $25 \%$ pensent que l'entrepreneuriat est un rêve et seul 5\% qui pensent que c'est une impossibilité. Toutefois il faut préciser que même s'ils ne pensent pas à entreprendre actuellement, cela ne signifie pas forcément l'absence de l'intention mais plutôt que le processus de création n'a pas encore été déclenché.

Ainsi, on peut avancer que l'attitude envers la création d'entreprise s'explique par la perception de l'étudiant sur le monde de l'entrepreneuriat. Dès lors nous allons nous intéresser aux 3 éléments fondamentaux évoqués par Ajzen (1991), qui interviennent quant à l'intention entrepreneuriale, à savoir l'attitude entrepreneuriale, la faisabilité et la désirabilité.

\subsection{L'attitude entrepreneuriale}

Tout d'abord, il convient de mentionner que l'attitude entrepreneuriale dépend principalement de la perception de l'individu sur l'entrepreneuriat. De 
ce fait, $61 \%$ des étudiants interviewés pensent qu'être entrepreneur n'est pas inné en nous, mais plutôt on le devient. 32\% estiment qu'un entrepreneur est une personne qui ne trouve pas sa place dans le monde professionnel, et finit donc par la créer lui-même, en détectant une opportunité d'affaires. En effet, $43 \%$ des interviewés pensent que le processus entrepreneurial commence par une idée, qui se traduit en une stratégie, pour pouvoir la développer et assurer sa croissance afin de garantir la réussite du projet de création d'entreprise.

Par ailleurs, les étudiants interviewés pensent entreprendre afin de développer leurs potentiels à raison de $43 \%, 35 \%$ pour des raisons financières et $23 \%$ considèrent que la création d'entreprise relève de l'estime de soi. Aussi nous remarquons que $49 \%$ des étudiants interviewés désirent percevoir un salaire supérieur à 15000 dirhams. De plus, ils ajoutent que la source du salaire varie entre un salaire fixe et les revenus complémentaires, ceci dit, les jeunes étudiants pensent à entreprendre des activités en parallèle à leurs fonctions principales. Quant à l'approche genre, on remarque que les étudiantes sondées ( $40 \%$ de l'échantillon) favorisent au même niveau que les étudiants sondés les postes de responsabilité, à raison de $96 \%$. Ainsi, on peut avancer que la motivation principale des étudiants est l'aspect financier à raison de $89 \%$.

Toutefois, ils soulignent que la création d'entreprise au Maroc connaît des obstacles qui freinent l'évolution de la culture entrepreneuriale chez les jeunes étudiants. En effet, l'absence de la culture entrepreneuriale est le principal obstacle à raison de $31 \%, 26 \%$ des étudiants pensent qu'il y a un manque de motivation, $20 \%$ des obstacles sont d'ordre socio-économiques, $13 \%$ des obstacles psychologiques et $12 \%$ des obstacles relatifs à la prise de décision. Quant aux valeurs entrepreneuriales qui influencent l'acte de création d'entreprise sont principalement les valeurs de créativité et de responsabilité. Il faut aussi reconnaître que la conviction est un déterminant majeur de l'intention entrepreneuriale qui est considérée comme une autoefficacité de l'individu, qui le laisse présager la réussite de l'action qu'il compte entreprendre. 
Figure 4. Motivations et freins à l'entrepreneuriat chez les étudiants
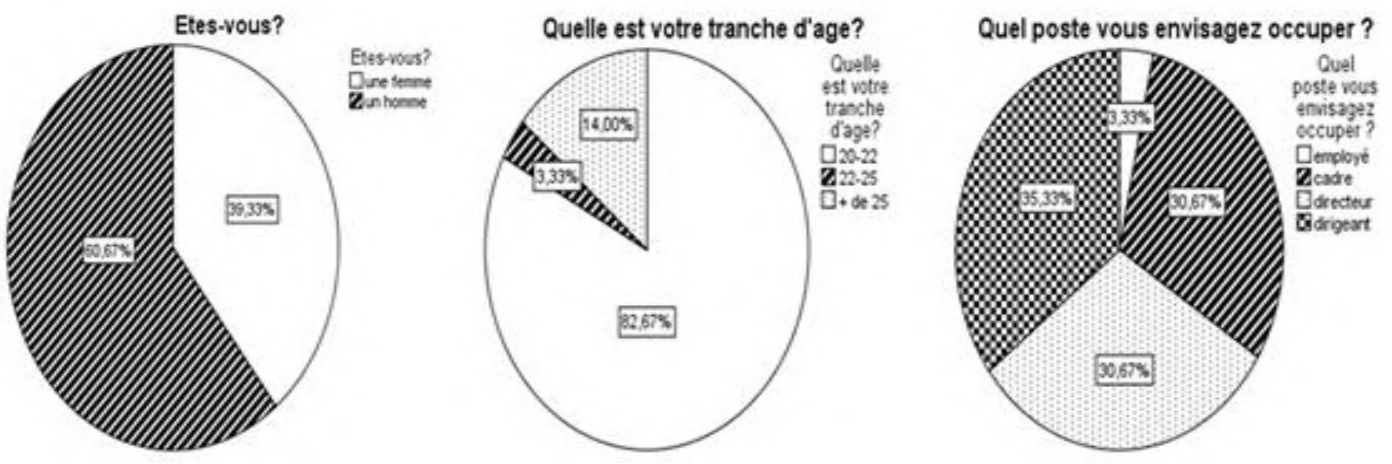

\subsection{La faisabilité}

L'importance des facteurs situationnels pour la décision entrepreneuriale est soulignée principalement par l'environnement social de l'individu. Toutefois, nous remarquons que $83 \%$ des étudiants interviewées connaissent des entrepreneurs. $68 \%$ pensent que l'expérience des entrepreneurs de leur environnement ont eu une expérience réussite, $30 \%$ que leur expérience était une bonne opportunité et seuls $2 \%$ ont pensé de l'expérience des entrepreneurs une erreur. Ainsi, nous remarquons que l'intention entrepreneuriale dépend principalement de la réussite du projet entrepreneurial de leur entourage. De plus, $88 \%$ des étudiants interviewés qui connaissent des entrepreneurs pensent à entreprendre. Cependant, le statut actuel de l'emploi - et ses changements - peut être considéré comme l'une des influences situationnelles les plus importantes. Le statut d'emploi est supposé affecter l'intention et la conviction.

Figure 5. Influence de l'environnement social sur l'intention entrepreneuriale
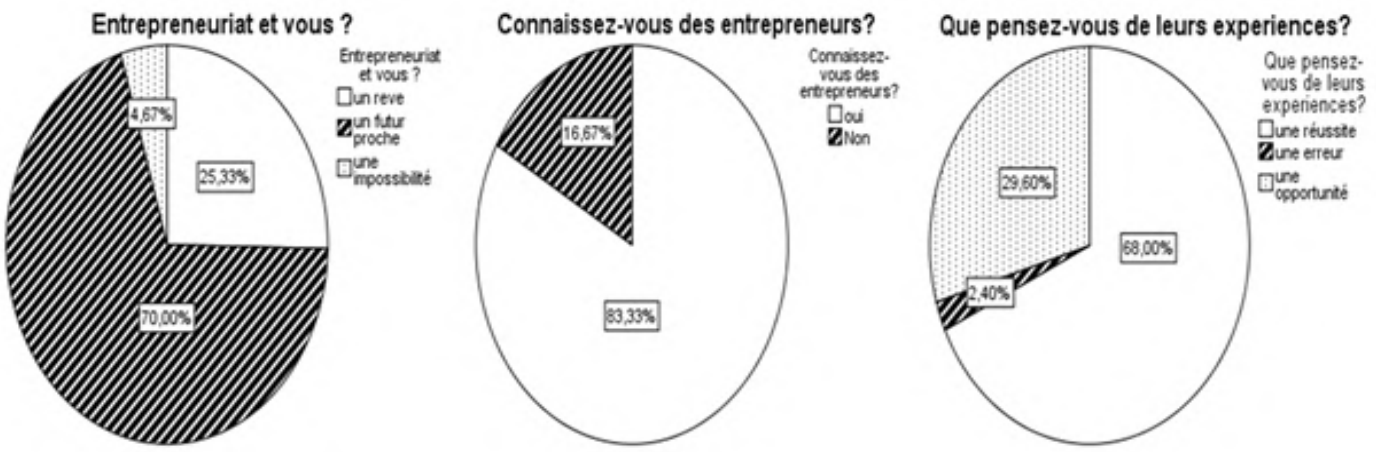

Parmi les facteurs situationnels, on peut considérer même les organisations mises en place par la politique du royaume dans la promotion de l'emploi et de la création d'entreprise, à savoir les organisations d'aide à la création d'entreprises. Malgré cet effort de promotion, notre échantillon reste 
un peu distant vis-à-vis de ces organisations. On remarque que seul $36 \%$ des étudiants interviewés les connaissent, et que seul $42 \%$ parmi eux ont l'intention de recourir aux services d'aide à la création d'entreprises. Alors que $30 \%$ des interviewés qui ne connaissent pas les incubateurs d'entreprises désirent bénéficier de leur services.

Il est à souligner que la formation en entrepreneuriat au niveau académique est importante, parce qu'elle oriente l'étudiant vers la détection des opportunités entrepreneuriales, la créativité et l'innovation. Plus de $40 \%$ des interviewés pensent que la formation en entrepreneuriat est importante voir très importante, contre $5 \%$ qui pensent qu'elle n'est pas du tout importante.

\subsection{La désirabilité}

Les perceptions de désirabilité sont fonction des attitudes envers l'entrepreneuriat et des normes sociales. L'intérêt que porte les étudiants pour la création d'entreprise est important dans l'échantillon étudié. L'analyse descriptive de cette variable démontre que $85 \%$ des étudiants consultés trouvent l'idée de créer leur affaire attractive. Selon eux, la création d'une entreprise leur permettrait de réaliser leurs rêves, de développer leurs potentiels et de mettre en œuvre leur créativité tout en ayant un travail intéressant qui engendrerait des bénéfices.

Le désir de créer une entreprise, porte principalement sur une nouvelle création à raison de $68 \%, 26 \%$ pensent à une affaire familiale et seuls $5 \%$ qui veulent reprendre une affaire déjà existante. Toutefois, la création d'une entreprise n'est pas une étape aisée. $47 \%$ des étudiants interviewés qui pensent entamer le processus de création d'entreprise, optent plutôt pour des fonds personnels, contre $32 \%$ qui favorisent l'emprunt auprès de la famille et $21 \%$ des interviewés qui pensent contracter un emprunt. Ce qui nous laisse considérer le problème de financement comme la principale limite quant à l'intention entrepreneuriale.

\section{Conclusion}

L'entrepreneuriat est un champ disciplinaire très important, puisqu'il favorise la création d'emploi et de la richesse. Cette création d'entreprise, ellemême, est précédée par l'intention de créer, que nous ne pouvons guère séparer de l'acte lui-même. Certes, toutes les intentions ne sont pas forcément susceptibles d'être concrétisées, cependant, elles représentent le meilleur élément de l'acte d'entreprendre. Nous avons tenté dans le cadre de ce travail de recherche, d'expliquer l'intention d'entreprendre à travers différents facteurs, essentiellement les facteurs liés aux attitudes entrepreneuriales, à la faisabilité de l'intention et sa désirabilité puisque l'influence la plus forte et la 
plus directe sur l'intention entrepreneuriale est attribuée à la conviction de l'individu.

Nos résultats dégagent, que les facteurs individuels et contextuels agissent positivement sur la vision entrepreneuriale qui sera traduite ultérieurement en une intention entrepreneuriale. L'analyse descriptive de l'attrait des étudiant(e)s pour la création d'entreprise dépend principalement de la conviction de l'individu et de la dimension temporelle qui impactent sur l'intensité de l'intention entrepreneuriale.

La recherche présentée ici résume et confirme de nombreuses recherches antérieures sur l'entrepreneuriat. Toutefois, il serait utile d'aller plus loin dans l'analyse des déclencheurs de de la décision d'action et aussi aux croyances, pour comprendre la suite du processus de la création d'entreprise et aussi repenser la sensibilisation à l'entrepreneuriat chez les jeunes étudiants.

\section{References:}

1. Ajzen, I. (1987). Attitudes, traits, and actions: dispositional prediction of behavior in personality and social psychology. Advances in experimental social psychology,20, 2-63.

2. Ajzen, I. (1991). The theory of planned behavior, Organizational Behavior and Human Decision Processes (pp. 179-1201). Vol. 50.

3. Belley, A. (1989). Opportunités d'affaires: objet négligé de la recherche sur la création d'entreprises (pp. 24-33). Revue PMO vol. $4, \mathrm{n}^{\circ} 1$.

4. Benavent, C. \& Verstraete, T. (2000). Entrepreneuriat et NTIC construction et régénération du Business-model. In Verstraete T. (ed), Histoire d'entreprendre- les réalités de l'entrepreneuriat. Paris. Management et Société.

5. Bird, B.J. (1988). Implementing entrepreneurial Ideas: The case for intention (pp.442-453). Academy of Management Review, 13(3).

6. Boyer, A. (1997). Le partage de l'intention, in : J.-P. DUPUY, P. LIVET, (sous la direction de), Les limites de la rationalité : rationalité, éthique et cognition (pp. 267-275). Tome 1, Editions La découverte.

7. Brockhaus, R.H. (1975). I-E locus of control scores as predictors of entrepreneurial intentions (pp.433-435). Academy of Management, Proceeding of the 35th Annual Meeting.

8. D'Amboise, G. (1989). La P.M.E. canadienne : situation et défis (pp.245). Institut de recherches politiques \& Les presses de l'Université Laval. Québec.

9. Davidsson, P. (2006). Nascent Entrepreneurship: Empirical Studies and Developments (pp. 1-76). Foundations and Trends in Entrepreneurship. 
10. Emin, S. (2003). L'intention de créer une entreprise des chercheurs publics : le cas français. Thèse en Sciences de Gestion. Université Pierre Mendés France, ESA, Grenoble.

11. Fayolle, A. (2002). Du champ de l'entrepreneuriat à l'étude $d u$ processus entrepreneurial: quelques idées et pistes de recherche. 6th CIFPME. Montréal.

12. Hernandez, E.M. (2001). L'entrepreneuriat. Approche théorique. L'Harmattan. Paris.

13. Hernandez, E.M. \& Marco, L. (2006). Entrepreneur et décision. De l'intention à l'acte. Editions ESKA. Paris.

14. Julien, P.A. \& Marchesnay, M. (1996). L'entrepreneuriat (pp.112). Edition Economica. Paris.

15. Learned, K.E. (1992). What happened before the organization? A model of organization formation (pp.39-48). Entrepreneurship Theory and Practice, 17(1).

16. Moreau, R. \& Raveleau, B. (2006). Les trajectoires de l'intention entrepreneuriale (pp.101-131). Revue Internationale PME, 19(2).

17. Raijman, R. (2001). Determinants of entrepreneurial intentions: Mexican immigrants in Chicago (pp.393-411). Journal of SocioEconomics.

18. Schumpeter, J. (1935). Théorie de l'évolution économique (pp.371). Edition Dalloz. Paris.

19. Shapero, A. (1975). The displaced, uncomfortable entrepreneur (pp.83-88). Psychology Today, vol. 9, n 6.

20. Shapero, A., Sokol, L. (1982). The social dimension of entrepreneurship (pp.72-90). In Kent C.A., Sexton D.L., Vesper K.H. (eds.), The Encyclopaedia of entrepreneurship, Englewood Cliffs NJ: Prentice Hall.

21. Tounès, A. (2003). L’intention entrepreneuriale. Une étude comparative entre des étudiants d'écoles de management et gestion suivant des programmes ou des formations en entrepreneuriat et des étudiants en DESS CAAE. Thèse en Sciences de Gestion. Université de Rouen.

22. Tounès, A. (2006). L’intention entrepreneuriale des étudiants : le cas français. Revue scientifique de gestion, $\mathrm{N}^{\circ} 219$.

23. Vallerand, R.J. (1994). Les fondements de la psychologie sociale. Boucherville, Québec :G.Morin. 


\section{Annexes}

\section{Annexe 1. Le questionnaire}

Première partie : Présentation

1. Etes-vous ?

- Une femme

- Un homme

2. Quelle est votre tranche d'âge ?

- 20-22

- $\quad 22-25$

- $\quad+$ de 25

3. Quel poste vous envisagez occuper?

- $\quad$ Employé

- $\quad$ Cadre

- Directeur

- Dirigeant

4. Quelle tranche de salaire souhaitez-vous percevoir?

- 3000-7000

- $\quad 7000-10000$

- $\quad 10000-15000$

- $\quad+$ de 15000

5. Quelle sera votre source du salaire ?

- $\quad$ Salaire fixe

- Commission

- $\quad$ Autres revenus complémentaires

Deuxième partie : Entrepreneuriat

6. Qu'est ce qu'entreprendre ?

- Une résolution

- Un engagement

- Une innovation

7. Qu'est ce qu'un entrepreneur ?

- Nait entrepreneur

- Devient entrepreneur

- $\quad$ Est une personne qui ne trouve pas sa place... donc il la fabrique

8. Quel est le processus d'entrepreneuriat ?

- Idée, stratégie, développement, croissance

- Décision, rationalité, opportunité, réactivité, organisation

- Vision, but, mission, plan 
9. Quelles sont les principales valeurs entrepreneuriales ?

\begin{tabular}{|l|l|l|l|l|l|}
\hline $\begin{array}{l}\text { Valeurs } \\
\text { entrepreneuriales }\end{array}$ & $\begin{array}{l}\text { Pas du tout } \\
\text { d'accord }\end{array}$ & $\begin{array}{l}\text { Pas } \\
\text { d'accord }\end{array}$ & Neutre & D'accord & $\begin{array}{l}\text { Tout à fait } \\
\text { d'accord }\end{array}$ \\
\hline $\begin{array}{l}\text { Valeurs } \\
\text { d'autonomie de }\end{array}$ & & & & & \\
\hline $\begin{array}{l}\text { Valeurs de } \\
\text { créativité }\end{array}$ & & & & & \\
\hline $\begin{array}{l}\text { Valeurs desponsabilité } \\
\text { respons de }\end{array}$ & & & & & \\
\hline $\begin{array}{l}\text { Valeurs } \\
\text { solidarité }\end{array}$ & & & & \\
\hline
\end{tabular}

10. Selon vous, quels sont les obstacles face à l'entrepreneuriat ?

- $\quad$ Socioéconomique

- Psychologique

- $\quad$ Absence de culture entrepreneuriale

- Motivation

- $\quad$ Capacité de prendre des décisions

11. Selon vous, qu'est-ce qui vous pousse à entreprendre ?

- $\quad$ Argent

- $\quad$ Estime de soi

- Développement du potentiel

12. Quelles sont les attitudes et capacités à favoriser chez un entrepreneur ?

\begin{tabular}{|l|l|l|l|l|l|}
\hline Attitudes et capacités & $\begin{array}{l}\text { Pas du tout } \\
\text { d'accord }\end{array}$ & $\begin{array}{l}\text { Pas } \\
\text { d'accord }\end{array}$ & Neutre & D'accord & $\begin{array}{l}\text { Tout à fait } \\
\text { d'accord }\end{array}$ \\
\hline Innovation & & & & & \\
\hline Prise de risque & & & & & \\
\hline Optimisme à à & & & & & \\
\hline Confiance en soi de & & & & \\
\hline $\begin{array}{l}\text { Tolérance } \\
\text { l'ambigüité et àncertitude }\end{array}$ & & & & & \\
\hline $\begin{array}{l}\text { Argent commesure } \\
\text { performance }\end{array}$ & & & & & \\
\hline Tolérance au stress & & & & & \\
\hline $\begin{array}{l}\text { Besoin } \\
\text { d'accomplissement }\end{array}$ & & & & & \\
\hline Besoin d'autonomie & & & & & \\
\hline $\begin{array}{l}\text { Habilité à reconnaître } \\
\text { une opportunité }\end{array}$ & & & & & \\
\hline Passion & & & & & \\
\hline Leadership & & & & & \\
\hline $\begin{array}{l}\text { Capacités } \\
\text { relationnelles }\end{array}$ & & & & & \\
\hline $\begin{array}{l}\text { Organisation } \\
\text { gestion de temps }\end{array}$ & & & & & \\
\hline Compétitivité & & & & & \\
\hline
\end{tabular}


13. Quelles sont les compétences en gestion nécessaires chez un entrepreneur ?

\begin{tabular}{|l|l|l|l|l|l|}
\hline & $\begin{array}{l}\text { Pas du tout } \\
\text { d'accord }\end{array}$ & $\begin{array}{l}\text { Pas } \\
\text { d'accord }\end{array}$ & Neutre & D'accord & $\begin{array}{l}\text { Tout à fait } \\
\text { d'accord }\end{array}$ \\
\hline $\begin{array}{l}\text { Gestion des } \\
\text { opérations da }\end{array}$ & & & & & \\
\hline $\begin{array}{l}\text { Gestion } \\
\text { trésorerie }\end{array}$ & & & & \\
\hline Marketing et vente & & & & & \\
\hline Gestion du personnel & & & & & \\
\hline $\begin{array}{l}\text { Résolution des } \\
\text { problèmes }\end{array}$ & & & & & \\
\hline Négociation & & & & & \\
\hline
\end{tabular}

14. Entrepreneuriat et vous

- Un rêve

- Un futur proche

- Une impossibilité

Troisième partie : Intentions entrepreneuriales

15. Connaissez-vous des entrepreneurs?

- Oui

- Non

16. Que pensez-vous de leurs expériences ?

- Une réussite

- Une erreur

- Une opportunité

17. Avez-vous déjà pensez à entreprendre ?

- Oui

- Non

18. Si oui, pour quelles raisons ?

- $\quad$ Estime de soi

- Argent

- Développement du potentiel

19. Dans le cas où vous voulez vous lancer, quelles seront les origines de vos fonds d'investissement?

- $\quad$ Fonds personnel

- $\quad$ Emprunt auprès de la famille

- $\quad$ Emprunt bancaire

20. Dans le cas où vous vous décidez de lancer votre propre entreprise, ca sera :

- Une affaire familiale

- Une reprise

- Une nouvelle création 
21. Connaissez-vous des associations d'aide à la création d'entreprise (incubateurs d'entreprise)?

$-\quad$ Oui
Non

22. Pensez-vous avoir recours à ce type d'association

- Oui

- Non

23. Avez-vous déjà eu une expérience en gestion d'équipe ?

- Oui

- Non

24. Est-il nécessaire de bénéficier d'une formation en entrepreneuriat?

- $\quad$ Pas du tout important

- Peu important

- Neutre

- Important

- Très important

25. Pouvez-vous prendre des engagements à long terme et travailler en vue d'atteindre des objectifs lointains?

- $\quad$ Oui

26. L'argent est il un objet de grande motivation pour vous?

- Oui

- Non

Annexe 2 : Les résultats

Première partie : Présentation
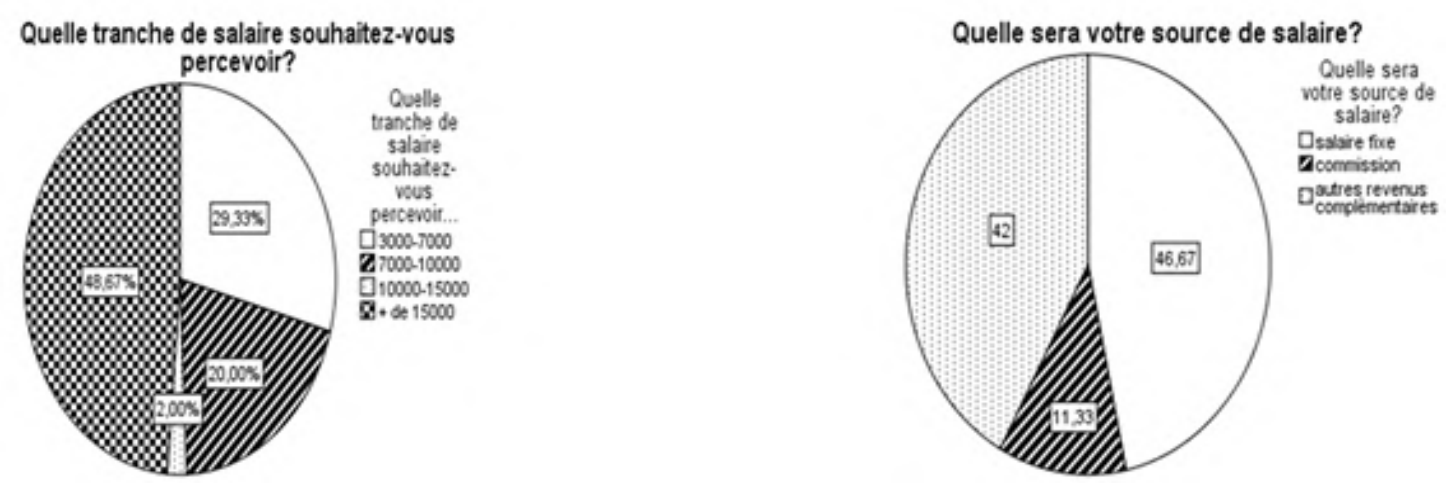

Deuxième partie : Entrepreneuriat 


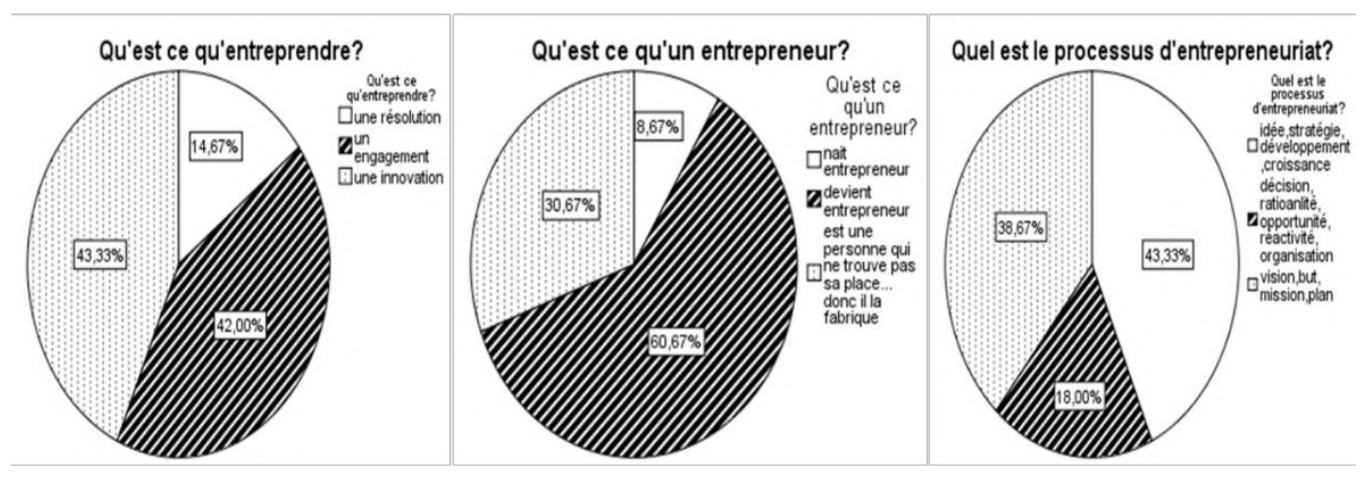

Les principales valeurs entrepreneuriales
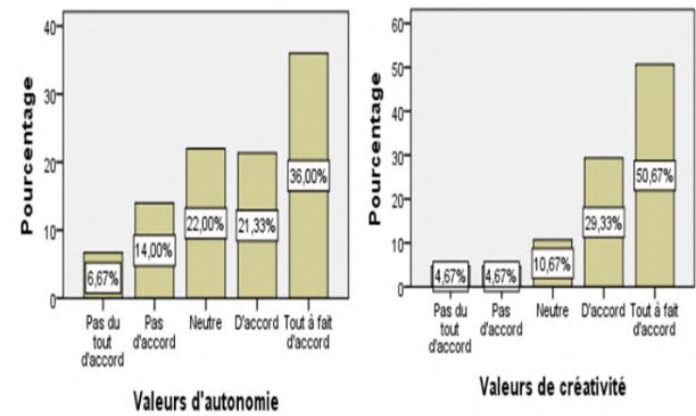

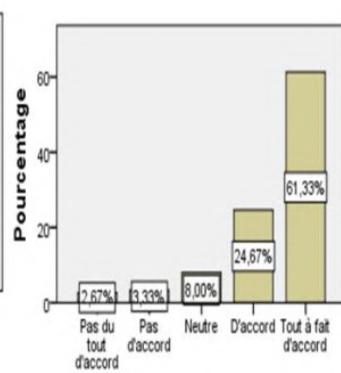

Valeurs de responsabilité

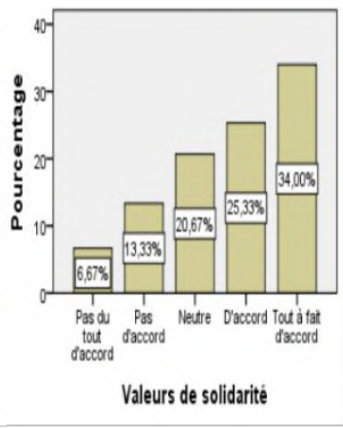


Les attitudes et les capacités à favoriser chez un entrepreneur
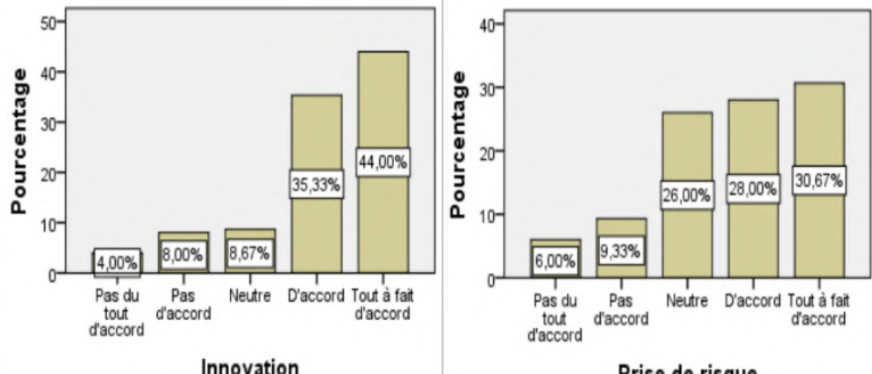

Prise de risque

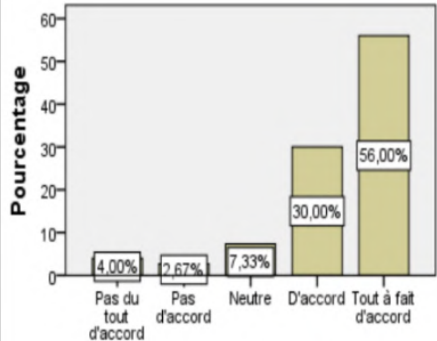

Confiance en sol

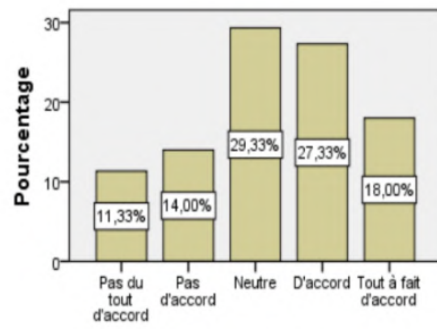

Tolérance au stress

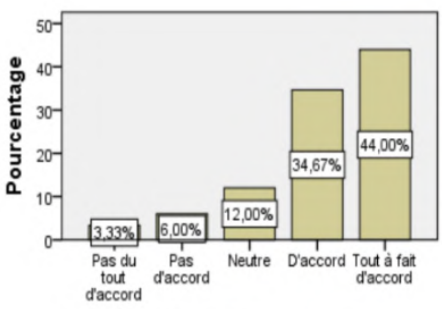

Habilité à reconnaitre une opportunité
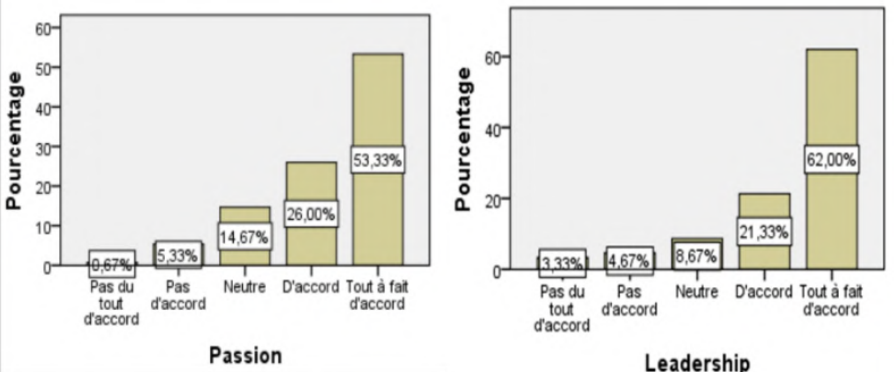

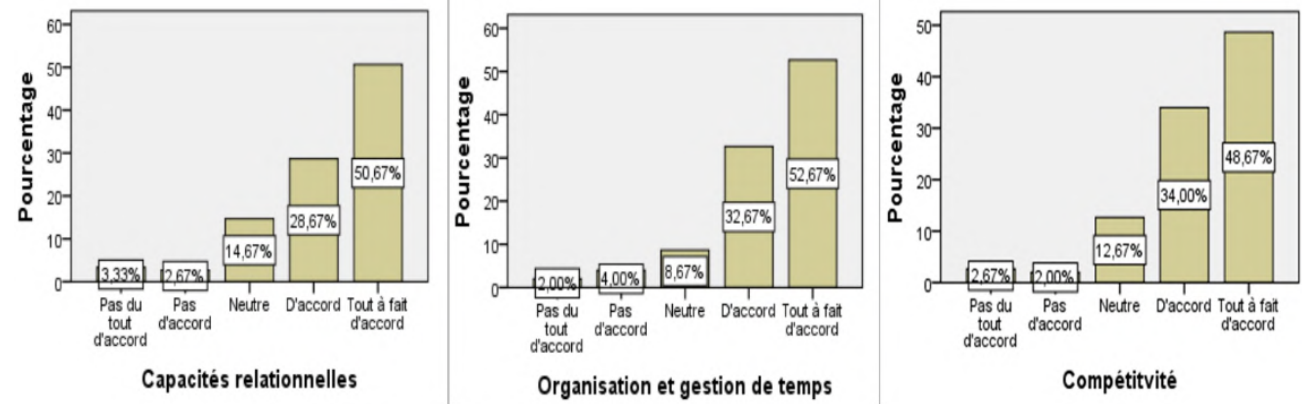

Les compétences en gestion nécessaires chez un entrepreneur
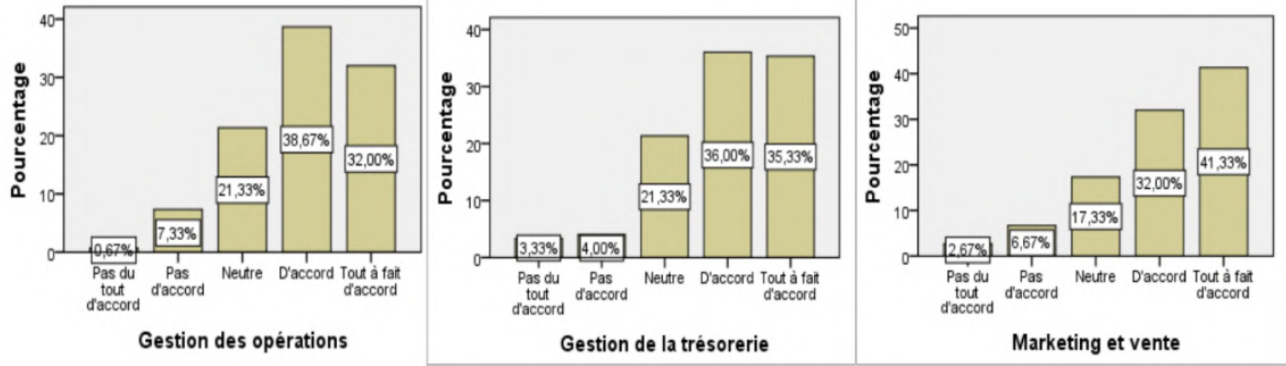

Gestion des opérations
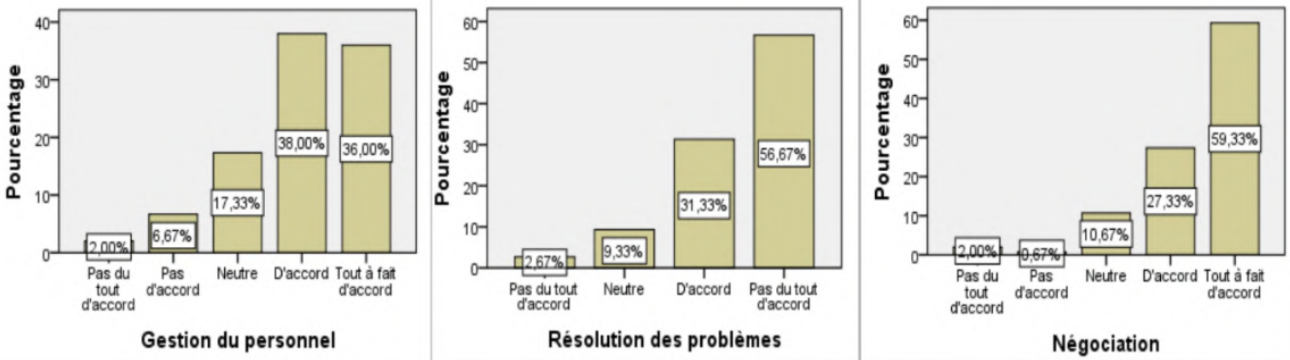

Troisième partie : Intentions entrepreneuriales
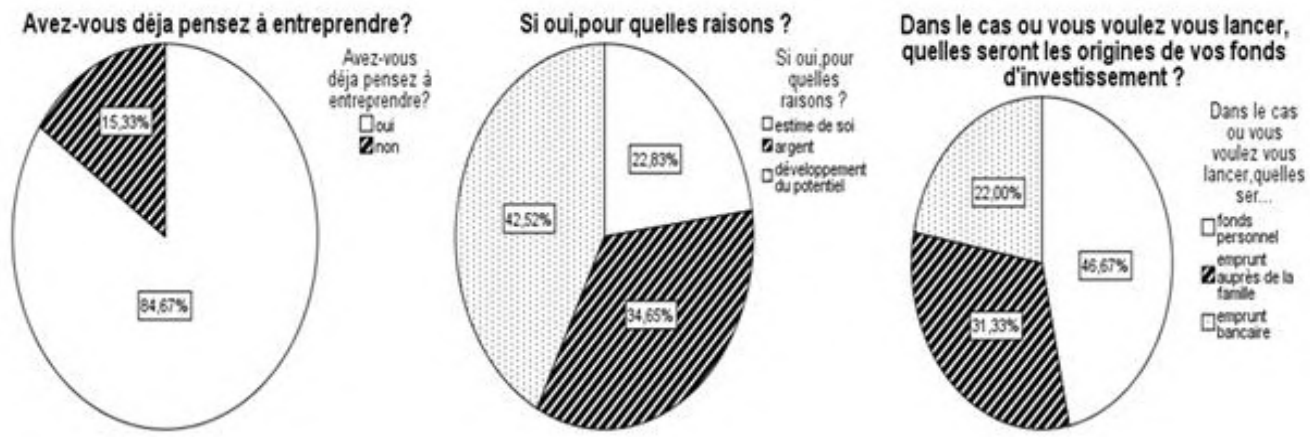
Dans le cas ou vous vous décidez de lancer votre propre entreprise,ca sera:

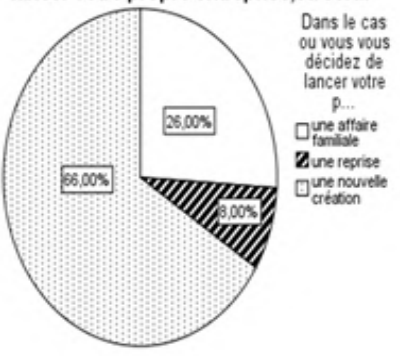

Avez-vous eu une formation en

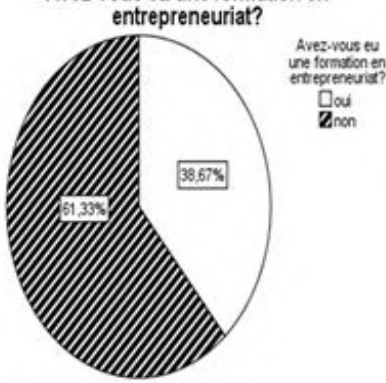

L'argent est il un objet de gande motivation

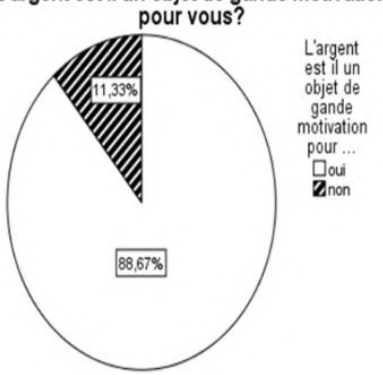

Connaissez-vous des associations d'aide à la creation d'entreprise(incubateurs

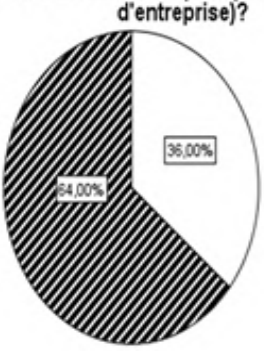

Connaissez.
vous des

associations

taide a la

cteat...

$\mathrm{Z}_{\operatorname{mon}}$

Est-ill nécessaire de béneficier d'une formation en entrepreneuriat?

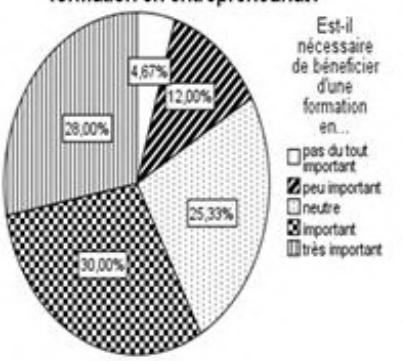

nécessait

de bénefcier

formation

apos dutos

Zpeu inportist

Dnetue

Iimpotart

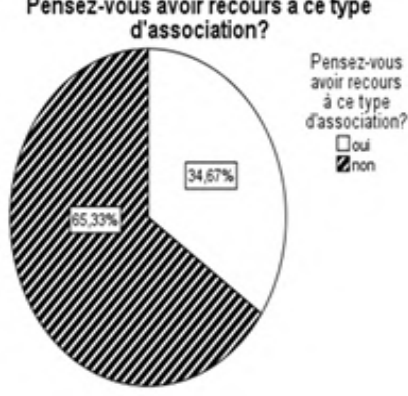

Pouvez-vous prendre des engagements à long terme et travailler en vue d'atteindre des objectifs lointains?

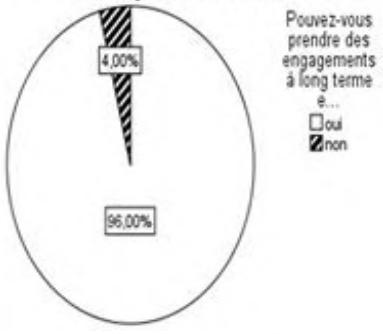

Quel est votre niveau d'étude?

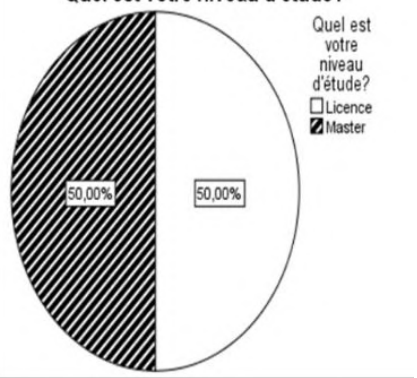

